\title{
ARBOL DE LA LLANURA ALUVIAL AMAZÓNICA Maquira coriacea (Karsten) C.C. Berg: ASPECTOS DE ECOLOGÍA Y MANEJO ${ }^{1}$
}

\section{Gustav Nebel ${ }^{2}$}

\section{RESUMEN}

Maquira coriacea es un árbol de valor comercial que se encuentra a lo largo y ancho de los bosques de la llanura aluvial inundable de la Amazonía. En este documento se describe su reproducción, su patrón de dispersión y otros atributos sobre la biología y ecología de su población. Se observaron densidades de hasta 14 individuos por hectárea con DAP $>10 \mathrm{~cm}$, con un área basal de $2 \mathrm{~m}^{2} / \mathrm{ha}$ y un volumen de $38 \mathrm{~m}^{3} / \mathrm{ha}$. El principal periodo de fructificación coincidió con la inundación anual, pero también se produjeron frutos en otros periodos del año. Se encontró regeneración natural con densidades de hasta $150 \mathrm{ind} / \mathrm{m}^{2}$ concentrados alrededor de los árboles madre; sin embargo, su permanencia fluctúa a lo largo del año por la mortalidad causada por las inundaciones y la marchitez en los periodos secos. Con el fin de desarrollar modelos de (1) relación altura-diámetro y (2) incremento de diámetro, se usaron principalmente datos de crecimiento de nueve parcelas de muestreo de una ha. Estas relaciones indican que el límite óptimo de diámetro de apeo para obtener el máximo volumen de producción es de 115-130 cm de DAP, los cuales pueden ser obtenidos en 135-300 años. Debido a que se ha observado producción de semillas en individuos muchos más pequeños, tales límites diamétricos no deberían ser desventajosos para la regeneración.

Palabras clave: Zonas húmedas, autoecología, reproducción, regeneración, dispersión, crecimiento, silvicultura, triplay.

1 Traducción del original en inglés "The amazon flood plain forest tree Maquira coriacea (Karsten) C.C. Berg: aspects of ecology and management".

2 Royal Veterinary and Agricultural University. Department of Economics and Natural Resources. Unit of Forestry, Rolighedsvej 23, 1958 Frederiksberg C., Denmark. Phone: + 45352822 32; Fax: 45352826 71. E-mail: gne@kvl.dk 


\begin{abstract}
Maquira coriacea is a commercial tree species which can be found throughout the Amazon flood plain forests. Its stocking, reproduction, dispersion pattern, and other attributes of population biology and ecology will be described in this paper. Densities up to $14 /$ ha of individuals $\geq 10 \mathrm{~cm} \mathrm{DBH}$ were observed, corresponding to a basal area of $2 \mathrm{~m}^{2} /$ ha and a volume of $38 \mathrm{~m}^{3} / \mathrm{ha}$. The main period of fruiting coincided with the annual flooding, but fruits were also produced in other parts of the year. Seedlings with densities up to $150 / \mathrm{m}^{2}$ were concentrated around mother trees, and the stocks fluctuated much over the year due to mortality caused by flooding and wilting in dry periods. Growth data mainly from nine one-hectare permanent sample plots were used to develop models of (1) the height - diameter relationship and (2) diameter increment. These relationships indicate an optimal felling limit for maximum volume production of 115-130 cm DBH, which can be obtained in 135-300 years. Since seed production has been observed in much smaller individuals, such diameter limits should not be detrimental to regeneration.
\end{abstract}

Key words: Peru, wetlands, autecology, reproduction, regeneration, dispersion, growth, silviculture, plywood.

\title{
1. INTRODUCCION
}

Los bosques húmedos tropicales se caracterizan por su compleja estructura y ecología; factores que a menudo complican el manejo del bosque. Recientemente, se han reforzado los esfuerzos para conocer a mayor profundidad la ecología de las especies arbóreas (por ej. Hubell y Foster, 1986; Bawa et al., 1990; Gómez-Pompa y Burley, 1991; Hubell 1995; Whitmore, 1995), muchos investigadores han descrito aspectos de la autoecología de las especies arbóreas del bosque tropical desde varios puntos de vista y usando diferentes métodos (por ej. Henriques y Sousa, 1989; Peters, 1990; Clark y Clark, 1992; McCormick, 1995; Zagt, 1997).

Este estudio se concentra en una especie arbórea maderable comercial que crece en los bosques de las llanuras aluviales inundables peruanas. El objetivo es describir aspectos de su biología y ecología y proporcionar información útil para su manejo. 


\section{MATERIALES Y METODOS}

El estudio de Maquira coriacea (Karsten) C.C. Berg se basa en los datos de campo de los bosques de la llanura aluvial inundable cercana a la localidad de Jenaro Herrera ( $\left.4^{\circ} 55^{\prime} \mathrm{S}, 73^{\circ} 44^{\prime} \mathrm{O}\right)$, localizada en el bajo Ucayali, en la selva baja de la Amazonía Peruana. Los aspectos generales de los bosques de las llanuras aluviales inundables peruanas, son descritos por Kvist y Nebel (1999), mientras que los detalles específicos del lugar de estudio fueron proporcionados por Nebel et al. (1999a, 1999b, 1999c, 1999d).

\subsection{Recolección de datos}

I. En nueve parcelas permanentes de muestreo de una hectárea (Nebel et al., 1999a) se registraron todos los individuos con DAP igual o mayor a $10 \mathrm{~cm}$, mientras que en las sub-parcelas de 0,64 ha (Nebel et al., 1999b) se registraron los individuos a partir de $1,5 \mathrm{~m}$ de altura hasta $10 \mathrm{~cm}$ de DAP. Las nueve parcelas de una hectárea fueron equitativamente distribuidas en los tres tipos de bosque de restinga alta, restinga baja y tahuampa, mientras que las sub-parcelas de sotobosque fueron establecidas solamente en los bosques de restinga. Después de establecer las parcelas, a fines de 1993, se llevó a cabo el apeo a dos intensidades. En cada tipo de bosque se dejó una parcela sin intervenir, mientras que las dos parcelas restantes fueron sometidas a un apeo moderado e intensivo, respectivamente. Todos los individuos fueron medidos nuevamente en 1994, 1995 y 1997. El crecimiento se registró en 1995 y 1997. Los registros incluyeron la medición del DAP, las coordenadas y la altura total estimada (Nebel et al., 1999a). Los datos de M. coriacea se usan para describir (1) su posición en los diferentes tipos de bosque, (2) su estructura poblacional y (3) su dinámica poblacional. Se consignaron un total de 432 registros de crecimiento de 147 individuos de $M$. coriacea.

II. A fines de 1994, se evaluó la presencia de plántulas de M. coriacea (altura < 1,5 $\mathrm{m})$, tanto en las parcelas de restinga alta como de restinga baja (Censo I). Dentro de las sub-parcelas de 20x20 m se estimaron las densidades de plántulas. Estos datos son usados para evaluar la dispersión de las plántulas y para evaluar el éxito de la regeneración.

III. A fines de 1996, se registraron en las parcelas de una hectárea (Censo I) los individuos de $M$. coriacea previamente no registrados. Sin embargo, solamente 
fueron registrados los individuos de las parcelas de los bosques de restinga alta, $\mathrm{y}$ de dos de las tres parcelas ubicadas en el bosque de restinga baja. Se midieron el DAP y la altura total. Se registraron un total de 312 individuos. Los datos son usados para analizar los patrones de dispersión.

IV. A fines de 1996 y a comienzos de 1997, se establecieron, en el bosque de restinga alta, parcelas permanentes de muestreo adicionales. Las parcelas fueron formadas como transectos de $40 \mathrm{~m}$ de ancho y una longitud total de $2,4 \mathrm{~km}$, de modo que cubrían un área de 9,6 ha. Se registró un total de 444 individuos de $M$. coriacea con alturas por encima de $1,5 \mathrm{~m}$. Los registros incluyeron las mediciones del DAP, las coordenadas y las alturas total y comercial. Se seleccionaron 80 individuos que representaban el rango total del diámetro y, mensualmente, durante el periodo de marzo 1997 a mayo 1998, se evaluó su fructificación. Los datos son usados para describir la relación altura - DAP y los patrones de fructificación.

V. Antes y después de la inundación, en 1998, se evaluó la cantidad de plántulas en las parcelas permanentes de muestreo establecidas en cuatro direcciones perpendiculares, lejos de los fustes de cinco árboles madre aislados de M. coriacea. En cada dirección se establecieron 25 parcelas con una longitud de $2 \mathrm{~m}$. Se consideraron segmentos de cinco parcelas y, empezando desde los más cercanos al fuste del árbol, las parcelas tuvieron anchos de 1, 2, 3, 4 y $5 \mathrm{~m}$ por cada segmento. Los datos son usados para evaluar la cantidad de plántulas en relación a la distancia desde los árboles padres.

\subsection{Análisis de datos}

Los valores de importancia de la especie (IVIs) para el estrato superior y el sotobosque fueron calculados mediante la sumatoria de las densidades relativas, frecuencias relativas y dominancias relativas de la especie. El método concuerda con Curtis y McIntosh (1950, 1951; fórmula dada por Nebel et al., 1999a).

Se evaluaron los patrones de dispersión de los individuos de $M$. coriacea pertenecientes al estrato superior y al sotobosque en los bosques de restinga alta y baja. Con el fin de cuantificar el grado de asociación a diferentes niveles, se calculó el índice de dispersión de Morisita para diversos tamaños de parcelas cuadradas (de 5, 10 y $20 \mathrm{~m}$ de longitud de lado, respectivamente). 


$$
I=\frac{\sum_{i=1}^{q} n i \cdot(n i-1)}{N \cdot(N-1)} \cdot q
$$

Donde $\mathrm{n}_{1}, \ldots \mathrm{n}_{\mathrm{q}}$ son los números de individuos observados en cada parcela cuadrada y $\mathrm{N}$ es el número total de individuos observados. La partida de la aleatorización $(\mathrm{I}=1,0)$ fue evaluada con la prueba de F (Greig-Smith, 1983). Asimismo, la distribución de las plántulas en cada tipo de bosque fue examinada usando un cuadrante de $20 \mathrm{~m}$ de longitud. Para evaluar el impacto de los tratamientos de apeo (Censo I), se analizaron posteriormente y en forma separada los datos de las parcelas sin intervenir. De esta manera, se determinó si el establecimiento y la sobrevivencia de las plántulas habían sido alterados, lo que podría resultar en un patrón de dispersión de plántulas distorsionado luego de ser comparado con las condiciones originales del rodal.

Los volúmenes se refieren al fuste y son calculados usando un factor de forma de 0,5, determinado por Cannell (1984) para algunas especies arbóreas tropicales.

\subsection{Distribución, morfología, propiedades de la madera y extracción}

El género Maquira (Moraceae) comprende cuatro especies neotropicales presentes principalmente en la parte norte de Sudamérica, las cuales se encuentran en la cuenca amazónica. El hábitat de $M$. coriacea son los bosques de la llanura aluvial inundable a lo largo y ancho de la cuenca del Amazonas, la cuenca del Orinoco y la cuenca del Alto río Paraguay, mientras que las otras especies se encuentran generalmente en bosques no inundables (Berg, 1972; Berg y Rosselli, 1996). M. coriacea es conocida en Perú como capinurí (Gentry, 1993) y juntamente con M. sclerophylla (Ducke) C.C. Berg como muiratinga o capinurí en Brasil (Chichignoud et al., 1990).

M. coriacea es un árbol de grandes aletas que alcanza una altura de $45 \mathrm{~m}$. Tiene un tronco cilíndrico de corteza lisa y ramas auto-desprendibles conspicuas que alcanzan diámetros de 1,3 m a más. Látex de color crema presente en la madera, corteza y hojas. Es dioica con flores masculinas (4-10 mm en diámetro), en manojos de 1-6, mientras que las flores femeninas se presentan solas o en pares, formando frutos elipsoidales o subglobosos de cerca de $1 \mathrm{~cm}$ de diámetro (Berg, 1972; Gentry, 1993). 
La madera de $M$. coriacea es de color blanco a marrón amarillento con un peso verde de $1050 \mathrm{~kg} / \mathrm{m}^{3}$ y un peso específico de $470 \mathrm{~kg} / \mathrm{m}^{3}$ al $12 \%$ de contenido de humedad. La contracción tangencial es de 6,7 a 9,4\%, mientras que la contracción radial está en el rango de 3,4 a $4,2 \%$. Es fácil de aserrar pero crea una superficie lanosa y el alto contenido de sílice desafila las sierras. Se puede laminar mediante torneado o rebanado a temperatura ambiente. La madera puede ser secada en dos días con un ligero riesgo de alabeo y rajaduras. Es de pobre durabilidad natural y, generalmente, se usa en la fabricación de madera contrachapada y en algunos otros productos de madera de poco valor comercial (Texeira et al., 1988; Chichignoud et al., 1990).

Ros-Tonen (1993) considera a M. coriacea como una de las especies más importantes extraídas de los llanos inundables de Brasil. Junto con Ceiba pentandra (L.) Gaertner y Virola surinamensis (Rolander) Warburg, es una especie de gran importancia para la producción de láminas y madera contrachapada en Brasil, aportando un ingreso de divisas del orden de 178 millones de dólares en 1989. En el departamento peruano de Loreto, $M$. coriacea es utilizada en la industria de la madera contrachapada. Según datos no publicados del Banco Central de Reserva del Perú, esta especie se hizo cargo de cerca de $2500 \mathrm{~m}^{3}(10 \%)$ de la producción de madera contrachapada en 1996, mientras que el $90 \%$ restante se produjo a base de $C$. pentandra (Kvist y Nebel, 1999).

\section{RESULTADOS}

\subsection{Reproducción}

Los árboles femeninos de $M$. coriacea pueden tener una prolongada y abundante fructificación. Un estudio fenológico indicó periodos bianuales de fructificación. El período entre diciembre y abril (aproximadamente) rinde la mayor cantidad de frutos y, parcialmente, coincide con el periodo de inundación anual (marzo a mayo). El otro período de fructificación es de junio a agosto y es menos vigoroso. Se observó fructificación en árboles de $40 \mathrm{~cm}$ de DAP a más. Solamente el 32\% de los individuos de esta categoría de tamaño no presentó fructificación. Igualmente, Berg (1972) acotó que probablemente las especies florecen a lo largo de todo el año y Pekka Soini (comunicación personal) informó que $M$. coriacea, en la Reserva Nacional PacayaSamiria, tiene características ecológicas relevantes, ya que su abundante y prolonga- 
da producción de frutos es una importante fuente de alimento para mamíferos y aves mayores.

Los animales mayores comen el fruto de $M$. coriacea. Sin embargo, a juzgar por la presencia de las plántulas, la gran mayoría de frutos queda por debajo de las copas de los árboles madre y solamente una pequeña proporción tiene una mayor dispersión (Figura 2). En este caso, las plántulas se presentan típicamente en agrupaciones de 20-50 individuos, lo que significa que pudieron ser dejados caer en el lugar por los animales al descansar o defecar. Los frutos que caen durante la época de inundación germinan al mermar el agua y pueden formar alfombras de plántulas con densidades de hasta $150 / \mathrm{m}^{2}$ por debajo de la copa de los árboles madre (Figura 2). La depredación de la semilla posterior a la etapa de dispersión puede ser alta y, probablemente, se debe a roedores. Son raros los ataques de pestes a las plántulas, pero largos periodos de sequía pueden causar la muerte. Casi todas las plántulas que sobreviven hasta la inundación perecen debido a ésta (Figura 2). Observaciones preliminares de un experimento sobre la exposición de plántulas a diferentes períodos de inundación indican que ellas empiezan a morir después de un mes de total inundación.

\subsection{Población, estructura y dinámica}

M. coriacea alcanzó considerables IVIs en todos los tipos de bosque, tanto en el estrato superior como en el sotobosque (Figura 3). En el estrato superior, la dominancia relativa fue más elevada que la densidad relativa, lo que indica que los individuos alcanzaron diámetros tan grandes como los diámetros de los promedios del bosque. Contrariamente, en el sotobosque, su dominancia relativa fue igual o menor que su densidad relativa, indicando alturas de árboles promedios o menores que los promedios.

El croquis de la posición de los árboles mostró que los individuos de $M$. coriacea en el sotobosque de la restinga alta, tienen un patrón de distribución heterogéneo y en grupos, mientras que no existe evidencia de patrón alguno en los individuos del estrato superior de la restinga alta o entre los relativamente pocos individuos en la restinga baja. Esto también fue evidente a partir de la proporción frecuencia relativa/ densidad relativa (Figura 3). Concordantemente, los cálculos del índice de Morisita para parcelas cuadradas de diferente longitud (Figura 4) mostraron una partida $(\mathrm{P}<0,001)$ de la dispersión al azar hacia la agrupación $\left(\mathrm{I}_{\delta}>1\right)$ en el sotobosque de restinga alta, aún cuando el estrato superior fue más o menos aleatoriamente espaciado $(\mathrm{P}>0,05)$. 
En el sotobosque de restinga alta, $M$. Coriacea presentó una curva aproximadamente exponencial negativa en valores de $\mathrm{I}_{\delta}$ al incrementar el área cuadrada de las parcelas (Figura 4). Aunque no se efectuaron los cálculos para las parcelas cuadradas extremadamente pequeñas, los resultados sugirieron un patrón de "puntos fuentes" con una alta densidad poblacional, rodeada de grupos más difusos de individuos con densidad declinando más o menos exponencialmente desde los centros de la población (Hubbell, 1979, 1980). Este patrón era particularmente pronunciado cuando se consideró a las parcelas no intervenidas en forma individual. Las plántulas, tal como se registraron en el Censo II, presentaron una pronunciada y altamente significativa $(\mathrm{P}<0,001)$ agregación al nivel de la parcela cuadrada de $20 \times 20 \mathrm{~m}\left(\mathrm{I}_{\delta}>6\right.$ y $>12$ para la restinga alta y baja, respectivamente).

Cuando analizamos separadamente, las plántulas de las parcelas no intervenidas no mostraron alejamiento significativo de la distribución aleatoria. Sin embargo, aquí se presentaron las plántulas en cantidades tan grandes que el índice de Morisita no sería apropiado para evaluar su patrón de distribución al nivel de las parcelas cuadradas de 20x20 m (Greig-Smith, 1983). En efecto, el promedio de la densidad de plántulas en las parcelas no tratadas en 1994 era de 10000 y 4000 por hectárea en la restinga alta y baja, respectivamente, comparado con la de 250 y 100 en las parcelas tratadas. Sin embargo, si la apertura de copas causa menos regeneración, no puede concluirse a partir de los resultados mencionados, ya que pueden haber sido decisivos otros factores, tales como la densidad de los árboles madre y la reducción temporal en la producción de frutos (debido a las perturbaciones).

La densidad disminuyó con el incremento de los diámetros, mostrando un patrón conocido como J invertida (Cuadro 1).

La relación altura - DAP fue diseñada usando la ecuación monomolecular o ecuación de Mitscherlich (Figura 5):

$$
\mathrm{h}=44,55 \cdot\left(1-0,9725 \cdot \mathrm{e}^{(-0,02361 * \mathrm{~d})}\right)
$$

donde $\mathrm{h}$ es la altura $(\mathrm{m})$ y d es el DAP $(\mathrm{cm})$.

La restinga alta contiene el mayor stock de $M$. coriacea, tanto en términos de densidad como de área basal, mientras que en la restinga baja el crecimiento (en área basal y volumen) en el periodo 1993-1997 fue el más alto (Cuadro 2). 
El mayor crecimiento en diámetro promedio anual de los árboles individuales se presentó en la clase $50-70 \mathrm{~cm}(2,2 \mathrm{~cm} / \mathrm{año})$, pero hubo una considerable variación (Cuadro 3). El área basal relativa promedio y el crecimiento en volumen, dentro de las clases de tamaño, varió de 5\% a 45\%. El tiempo en que los árboles alcanzan 130 cm de DAP es de 112 y 33 años, en función de las tasas promedio y máximo de crecimiento en DAP, respectivamente.

El incremento en diámetro de $M$. coriacea fue generalmente alto, pero varió considerablemente con el DAP. Usando la variable DAP se ajustaron dos modelos basados en la ecuación de Wykoff ( $c f$. Vanclay, 1994). El primer modelo se ajustó para los datos de las parcelas no tratadas, usando la variable DAP (3), mientras que el segundo modelo se fijó para los datos de las parcelas tratadas, usando de la misma manera, la variable DAP (4):

$$
\begin{aligned}
& \Delta d=\sqrt{\mathrm{e}^{\left(-9,521892+3,063883 \cdot \operatorname{In}(\mathrm{d})-0,000489 \cdot \mathrm{d}^{2}\right)}} \\
& \Delta d=\sqrt{\mathrm{e}^{\left(-9,480924+3,105072 \cdot \operatorname{In}(\mathrm{d})-0,000509 \cdot \mathrm{d}^{2}+15,346986 \cdot \mathrm{e}^{(-\sqrt{d})}\right.}}
\end{aligned}
$$

donde $\Delta \mathrm{d}$ es el incremento diamétrico en $\mathrm{cm}$ y d es el DAP en $\mathrm{cm}$. Las pruebas $\mathrm{F}$. Standard mostraron buen ajuste de todos los modelos $\left(\mathrm{R}^{2}=0,45\right.$ (2); 0,54 (3), $\mathrm{P}<0,0001$ ). El modelo (3) predice que el incremento anual corriente de DAP llega a su máximo alrededor de $55 \mathrm{~cm}$ DAP y es relativamente bajo para pequeños diámetros, resultando en un bajo incremento anual corriente en DAP que alcanza su máximo cerca de los $120 \mathrm{~cm}$ DAP (Figura 6a). El modelo (4) también predice que el incremento anual del DAP alcanza su máximo alrededor de los $55 \mathrm{~cm}$ DAP, pero tiene incrementos más altos a diámetros más pequeños, lo que resulta en un alcance más temprano y más pronunciado del incremento promedio anual alrededor de los $100 \mathrm{~cm}$ DAP (Figura 6b).

\section{DISCUSION}

La madera de $M$. coriacae es apropiada para la producción de contrachapados y se extrae en gran cantidad en toda la extensión de su hábitat natural, los llanos inundables 
de América del Sur tropical (Berg, 1972; Texeira et al., 1988; Chichignoud et al., 1990; Ros-Tonen, 1993). Es generalmente abundante, frecuente y dominante en los bosques de las llanuras aluviales inundables peruanas, aunque aparentemente prefiere los bosques de restinga de corta inundación y se convierte en menos dominante en los bosques de tahuampas de inundación más prolongada. La importancia total relativa (IVIs) de $M$. coriacea correspondió a 1,5-5\% de todas las especies del estrato superior y a $0,3-2,5 \%$ del sotobosque (Figura 3), lo cual es alto para una sola especie del bosque húmedo tropical. Aplicando un apropiado manejo, estos valores podrían ser manipulados tal vez hasta un punto más alto, si se quisiera.

Los estudios fenológicos del M. coriacea en 1997 y 1998 mostraron que la mayor fructificación tuvo lugar entre diciembre y mayo, coincidiendo con el periodo de inundación anual. Sin embargo, en 1997 los árboles también fructificaron de junio a agosto, mientras que en 1998 no se observó fructificación en este periodo, talvez debido a la inusual acrecencia de un periodo seco que impidió el desarrollo del fruto (Figura 1).

Estas observaciones están de acuerdo a Zerg (1972), quien sostuvo que la especie, probablemente, florece todo el año.

Similarmente, Pekka Soini (comun. personal) informo que $M$. coriacea en la Reserva Natural Pacaya Samiria es una fuente importante de alimento para mamíferos y grandes pájaros, debido a su prolongada y abundante producción de frutos. Desde que muchos otros árboles de la llanura aluvial inundable solamente fructifican después de la inundación, es importante contar con individuos en edad fértil de $M$. coriacea, como una fuente de alimento para pájaros y mamíferos.

El debilitamiento durante prolongados periodos de sequía y el ahogamiento relacionado con los periodos de mayor inundación, parecieron ser las principales causas de mortalidad, lo cual resulta en fluctuaciones en el tiempo del stock de plántulas (Figura 2). Debido a que las densidades de plántulas fueron claramente más elevadas debajo de la copa de los árboles padre, se pudo notar también restricciones en el espacio (Figura 2). A pesar de las fluctuaciones en tiempo y espacio con relación a la presencia de semillas y plántulas, se puede decir que, desde el punto de vista del manejo forestal, el patrón reproductivo es positivo. Parece que es posible esperar encontrar, en gran parte del año, abundante cantidad de plántulas localizadas cerca de los árboles semilleros. Sin embargo, las observaciones realizadas en nuestras parcelas tratadas sugieren que la germinación de las semillas o el establecimiento de las plántulas y el crecimiento pueden ser impedidos por la directa y repentina exposición a condiciones de mucha luz. En áreas con periodos de inundación más prolongados, 
donde mueren las plántulas, excepto en años con periodos cortos de inundación, plantar plántulas de mayor tamaño puede ser una manera de conservar la especie, puesto que la tolerancia a la inundación generalmente aumenta con el tamaño de los individuos (Gill, 1970). Desde el punto de vista del establecimiento de plántulas, es preferible llevar a cabo las operaciones de extracción justo después de la inundación a fin de permitir a las plántulas crecer antes de la siguiente inundación. Esto podría no estar de acuerdo con la ventaja técnica de apear los árboles justo antes de la inundación y permitir el transporte por flotación de las trozas hasta las triplayeras junto con otras especies flotantes. En los bosques estudiados se encontraron abundantes plantones mayores, pero éstos disminuyeron con la duración de la inundación (Cuadro 1). El objetivo del manejo debería orientarse a favorecer estos plantones, los cuales son abundantes en la restinga alta y donde el riesgo de la erosión genética sería menor si son favorecidos con éxito.

Los diferentes patrones de dispersión de $M$. coriacea en el sotobosque y el estrato superior de la restinga alta (Figura 4) pueden ser interpretados de varias maneras. La distribución en manchales de los individuos en el sotobosque puede reflejar una variación de condiciones microzonales y procesos biológicos cruciales en el establecimiento y sobrevivencia de las plántulas. La reducción en el agrupamiento de plántulas con el incremento en su tamaño puede ser indicador de un auto clareo, una mortalidad dependiente de la densidad o de la incompleta colonización del sitio (Hubbell, 1979, 1980; Greig-Smith, 1983; Henriques y Sousa, 1989). En la restinga alta, las condiciones de crecimiento fueron más o menos homogéneas en términos de drenaje y fertilidad del suelo, lo que implica que existen otros factores que causaron el agrupamiento de plantones. No obstante las condiciones de luz fueron en manchas, en la parcela en donde se llevó a cabo no había mayor tendencia hacia una dispersión aleatoria, dando lugar a condiciones más homogéneas de luz. Es más probable que la dispersión restringida de semillas sea la razón principal de la distribución por grupos dispersos de los plantones, los cuales, debido a la mortalidad dependiente de la densidad, desarrollaron a una dispersión más aleatoria entre todos los individuos mayores (Figura 4).

Los cálculos del crecimiento por clase diamétrica de $M$. coriacea (Cuadro 3) parecen ser una metodología apropiada para representar el crecimiento y rendimiento de las especies. Sin embargo, al compararlo con las predicciones de los modelos de crecimiento desarrollados (2) y (3), los resultados del crecimiento por clase diamétrica indican un desarrollo relativamente rápido, especialmente cuando se toma como base el máximo crecimiento. Los modelos combinados de incremento en diámetro y altura predicen el término del incremento promedio anual en volumen aproximadamente 
a los $130 \mathrm{~cm}$ DAP, cuando se usan los datos de las parcelas no tratadas (Figura 6c) y alrededor de $115 \mathrm{~cm}$ DAP en las parcelas tratadas (Figura 6d). Estos puntos también indican el límite óptimo del diámetro de apeo para la máxima producción en volumen, el cual se alcanza en aproximadamente 300 y 135 años, respectivamente. La considerable diferencia entre los dos modelos se debe principalmente al lento desarrollo de los individuos jóvenes en las parcelas no tratadas, lo que indica que los tratamientos de apeo pueden tener un efecto deseable sobre el crecimiento de $M$. coriacea. Así, el diámetro límite para la óptima producción en volumen está en el rango de 115-130 cm DAP, dependiendo del tipo de modelo utilizado, el cual es alcanzado en 135-300 años. Si estos límites son también económicamente óptimos, depende de otros factores. Debido a que se ha observado fructificación en individuos de $45 \mathrm{~cm}$ DAP, y que ésta puede ser abundante en árboles de 60-100 cm DAP, un diámetro límite en el rango de 115-130 cm DAP no debería tener repercusiones en la regeneración de la especie. Tal como se indicó mediante las tasas de máximo crecimiento (Cuadro 3), el desarrollo del crecimiento puede ser talvez aún mejor con los tratamientos óptimos de raleo.

El relativo alto stock de $M$. coriacea (Cuadro 2) es algo inusual en los bosques húmedos neotropicales ricos en especies, para una sola especie arbórea comercial (por ej. Lamprecht, 1989, 1993; Silva, 1989). Más aún, sus tasas de crecimiento fueron relativamente altas (Cuadro 2, Cuadro 3, Figura 6). Combinado con sus características reproductivas, las cuales son relativamente positivas desde el punto de vista del manejo forestal, implica que existen muchas opciones de manejo forestal. A pesar de la tendencia de las plántulas y plantones de crecer agrupados (Figura 2, Figura 4), la distribución horizontal y vertical de los individuos más grandes fue relativamente homogénea, lo cual es deseable para implementar un sistema silvicultural policíclico, especialmente en la restinga alta. La abundante y confiable regeneración, por otro lado, sería probablemente también suficiente para lograr el establecimiento de un sistema silvicultural monocíclico que se concentre en la especie. Cualquiera de estas propuestas serían factibles, dependiendo de los objetivos de la empresa. Sin embargo, el sistema policíclico puede ser más consistente con el mantenimiento de muchos otros servicios proporcionados por los bosques de la llanura inundable. Puesto que ellos están, por naturaleza, adaptados a las grandes perturbaciones (Foster et al., 1986; Salo et al., 1986; Foster, 1990a, 1990b; Worbes et al., 1992; Salo y Kalliola, 1993; Worbes, 1997), pueden probablemente sostener los disturbios ocasionados por la extracción maderera, especialmente los bosques de restinga de rápida respuesta. $M$. coriacea podría contribuir significativamente a la extracción maderera en tales sistemas. 


\section{RECONOCIMIENTOS}

Al Instituto de Investigaciones de la Amazonía Peruana (IIAP) y su estación de campo en el Centro de Investigaciones Jenaro Herrera (CIJH) por proporcionar gratamente sus instalaciones de campo y la logística. Al personal del CIJH y a la gente de Jenaro Herrera por aceptar contribuir a completar el trabajo de campo, especialmente a Manuel Isuiza, Héctor Vidaurre, Luis Freitas, Arístides Vásquez, Nitzen Saavedra, David Maytahuari, Francisco Cahique, Julio Irarica, Leandro Ruiz y Jaime Vásquez. Los fondos fueron proporcionados por la Agencia Danesa de Desarrollo Internacional (DANIDA) y el Centro para la Investigación Forestal Internacional (CIFOR).

\section{BIBLIOGRAFIA}

BAWA, K.S.; ASHTON, P.S.; NOR, S.M. 1990. Reproductive ecology of tropical forest plants: management issues. In: K.S. Bawa; M. Hadley (editors). Reproductive ecology of tropical forest plants. París: UNESCO. pp. 3-13.

BERG, C.C. 1972. Flora Neotropica. Olmedieae, Brosimaea (Moraceae). Monograph $N^{\circ} 7$. New York: Hafner Publishing Company. 229 pp.

BERG, C.C.; ROSSELLI, P.F. 1996. New taxa and combinations in Moraceae and Cecropiaceae from Central and South America. In: Novon, 6:230-252.

CANNELL, M.G.R. 1984. Woody biomass of forest stands. In: Forest ecology and management, 8: 299-312.

CHICHIGNOUD, M.; DEÓN, G.; DETEINNE, P.; PARANT, B.; VANTOMME, P. 1990. Tropical timber atlas of Latin America. Yokohama: ITTO/CTFT, $218 \mathrm{pp}$.

CLARK, D.A.; CLARK, D.B. 1992. Life history diversity of canopy and emergent trees in a neotropical rain forest. In: Ecological Monographs, 62(3): 315344.

CURTIS, J.T.; MCINTOSH, R.P. 1950. The interrelations of certain analytic and synthetic phytosociological characters. In: Ecology, 31(3): 435-455. 
1951. An upland forest continuum in the prairie-forest border region of Wisconsin. In: Ecology, 32(3): 476-496.

FOSTER, R.B. 1990a. Long-term change in the successional forests community of the Rio Manu floodplain. In: A.H. Gentry (editor). Four neotropical rainforests. New Haven: Yale University Press, pp. 565-572.

1990b The floristic composition of the Rio Manu floodplain forest. In: A.H. Gentry (editor), Four neotropical rainforests. New Haven: Yale University Press, pp. 99-111.

FOSTER, R.B.; ARCE, J.B.; WACHTER, T.S. 1986. Dispersal and the sequential plant communities in Amazonian Peru floodplain. In: A. Estrada; T.H. Fleming (editors). Frugivores and seed dispersal. Dordrecht: Dr. W. Junk Publishers. pp. 357-370.

GENTRY, A.H. 1993. A field guide to the families and genera of woody plants of northwest South America (Colombia, Ecuador, Perú) with supplementary notes on herbaceous taxa. Chicago: University of Chicago Press. 895 pp.

GILL, C.J. 1970. The flooding tolerance of woody species - a review. In: Forestry Abstracts, 31 (4): 671-688.

GÓMEZ-POMPA, A.; BURLEY, F.W. 1991. The management of natural tropical forests. In: A. Gomez-Pompa; T.C. Whitmore; M. Hadley (editors). Rain forest regeneration and management. Paris: UNESCO. pp. 3-18.

GREIG-SMITH, P. 1983. Quantitative plant ecology. In: Blackwell Scientific Publications. 359 pp.

HENRIQUES, R.P.B.; SOUSA, E.C.E.G. 1989. Population structure, dispersion and microhabitat regeneration of Carapa guianensis in Northeastern Brazil. In: Biotropica, 21 (3): 204-209.

HUBBELL, S.P. 1979. Tree dispersion, abundance, and diversity in a tropical dry forest. In: Science, 203 (4387): 1299-1309.

1980. Seed predation and the coexistence of tree species in tropical forests. In: OIKOS, 35: 214-229. 
1995. Toward a global research strategy on the ecology of natural tropical forests to meet conservation and management needs. In: A.E. Lugo; C. Lowe (Editors). Tropical forests: management and ecology. Berlin: SpringerVerlag. pp. 423-437.

HUBBELL, S.P.; FOSTER, R.B. 1986. Commonness and rarity in a neotropical forest: implications for tropical tree conservation. In: M. Soulé (editor). Conservation biology: science of scarcity and diversity. Sinauer, Sunderland. pp. 205-231.

KVIST, L.P.; NEBEL, G. A. 1999. Review of Peruvian flood plain forests: Ecosystems, inhabitants and resource use. Copenhagen: Royal Veterinary and Agricultural University, Department of Economics and Natural Resources, Unit of Forestry. Unpublished manuscript.

LAMPRECHT, $H$. Silviculture in the tropics. 1989. Tropical forest ecosystems and their tree species - possibilities and methods for their long-term utilization. Eschborn: GTZ, 296 pp.

1993. Silviculture in the tropical natural forests. In: L. Pancel (editor), Tropical forestry handbook. Berlin: Springer-Verlag. pp. 727-810.

MCCORMICK, J.F. 1995. A review of the population dynamics of selected tree species in the Luquillo experimental forest, Puerto Rico. In: A.E. Lugo; C. Lowe (editors). Tropical forests: management and ecology. Berlin: Springer Verlag. pp. 224-257.

NEBEL, G.; KVIST, L.P.; VANCLAY, J.K.; CHRISTENSEN, H.; FREITAS, L.; RUIZ, J. 1999a. Structure and floristic composition of flood plain forests in the Peruvian Amazon: I. Overstorey. Copenhagen: Royal Veterinary and Agricultural University, Department of Economics and Natural Resources, Unit. of Forestry. Unpublished manuscript.

NEBEL, G.; DRAGSTED, J.; VANCLAY, J.K. 1999b. Structure and floristic composition of flood plain forests in the Peruvian Amazon: II. The understorey of restinga forests. Copenhagen: Royal Veterinary and Agricultural University, Department of Economics and Natural Resources, Unit of Forestry. Unpublished manuscript. 
NEBEL, G.; KVIST, L.P.; VANCLAY, J.K.; VIDAURRE, H. 1999c. Forest dynamics in flood plain forests in the Peruvian Amazon: Effects of disturbance and implications for management and conservation. Copenhagen: Royal Veterinary and Agricultural University, Department of Economics and Natural Resources, Unit of Forestry. Unpublished manuscript.

NEBEL, G.; DRAGSTED, J.; SALAZAR, A.V. 1999d. Litter fall, biomass and net primary production in flood plain forest in the Peruvian Amazon. Copenhagen: Royal Veterinary and Agricultural University, Department of Economics and Natural Resources, Unit of Forestry. Unpublished manuscript.

PETERS, C.M. 1990. Population ecology and management of forest fruit trees in Peruvian Amazonia. In: A.B. Anderson (editor). Alternatives to deforestation: Steps toward sustainable use of the Amazon rain forest. New York: Columbia University Press. pp. 86-98.

ROS-TONEN, M.A.F. 1993. Tropical hardwood from the Brazilian Amazon. Saarbrücken - Fort Lauderdale: Verlag Breitenbach Publishers. 279 pp.

SALO, J.; KALLIOLA, R. 1993. Necesidades para el futuro en la selva baja peruana: Conservación e investigación cientifica. In: R. Kalliola; M. Puhakka; W. Danjoy (editors). Amazonia peruana. Vegetación húmeda trcopical en el llano subandino. Jyväskylä: PAUT/ONERN. pp. 235-250.

SALO, J.; KALLIOLA, R.; HÄKKINEN, I.; MÄKINEN, Y.; NIEMELÄ, P.; PUHAKKA, M.; COLEY, P.D. 1986. River dynamics and the diversity of lowland forest. In: Nature, 322:254-258.

SILVA, J.N.M. 1989. The behaviour of the tropical rain forest of the Brazilian Amazon after logging. Oxford: Oxford Forestry Institute. 302 pp.

TEIXEIRA, D.E.; SANTANA, M.A.E.; SOUZA, M.R. de. 1988. Amazonian timbers for the international market. In: ITTO Technical Series 1. Yokohama: IBDF/ ITTO. 94 pp.

VANCLAY, J.K. 1994. Modelling forest growth and yield. Applications to mixed tropical forests. Wallingford: CAB International. $312 \mathrm{pp}$. 
WHITMORE, T.C. 1995. Perspectives in tropical rain forest research. In: A.E. Lugo; C. Lowe (editors). Tropical forests: ecology and managements. Berlin: Springer- Verlag. pp. 397-407.

WORBES, M. 1997. The forest ecosystem of the floodplain. In: W.J. Junk (editor). The central Amazon floodplain Ecology of a pulsing system. Berlin: SpringerVerlag. pp. 223-266.

WORBES, M.; KLINGE, H.; REVILLA, J.D.; MARTIUS, C. 1992. On the dynamics, floristic subdivision and geographical distribution of várzea forests in Central Amazonia. In: Journal of Vegetation Science, 3: 553-564.

ZAGT, R.J. 1997. Tree demography in the tropical rain forest of Guyana. Series 3. Utrecht University, Tropenbos-Guyana. 251 pp. 
Cuadro 1. Distribución diamétrica del número de árboles.

\begin{tabular}{|c|c|c|c|}
\hline DAP (cm) & Restinga alta & Restinga baja & Tahuampa \\
\hline $0-9$ & 418 & 35 & -1 \\
$10-19$ & 18 & 5 & 6 \\
$20-29$ & 8 & 5 & 2 \\
$30-39$ & 6 & 2 & - \\
$40-49$ & 2 & 1 & 2 \\
$50-59$ & 2 & - & 1 \\
$60-69$ & 2 & 3 & 1 \\
$70-79$ & 1 & 4 & 1 \\
$80-89$ & 1 & - & 1 \\
$90-99$ & 1 & 1 & - \\
$110-119$ & 1 & - & - \\
$120+$ & 1 & - & - \\
\hline
\end{tabular}

1 Datos no disponibles.

Cuadro 2. Stock y crecimiento de Maquira coriacea en bosques de la llanura aluvial inundable.

\begin{tabular}{|l|r|r|r|}
\hline & $\begin{array}{r}\text { Restinga } \\
\text { alta }\end{array}$ & $\begin{array}{c}\text { Restinga } \\
\text { baja }\end{array}$ & Tahuampa \\
\hline Densidad (ind/ha) & 14,3 & 7,0 & 4,7 \\
Area basal (m² por ha) & 2,1 & 1,4 & 0,7 \\
Volumen del tronco (m ${ }^{3}$ por ha) & 37,9 & 23,8 & 11,1 \\
Diámetro (cm) correspondiente al área & 30,7 & 41,8 & 36,3 \\
basal promedio en pie & 0,085 & 0,087 & 0,025 \\
$\begin{array}{l}\text { Crecimiento promedio anual del área } \\
\text { basal (m²/ha/año) }\end{array}$ & 1,46 & 1,68 & 0,47 \\
$\begin{array}{l}\text { Crecimiento promedio anual de volumen } \\
\left(\mathrm{m}^{3} / \text { ha/año) }\right.\end{array}$ & & & \\
\hline
\end{tabular}




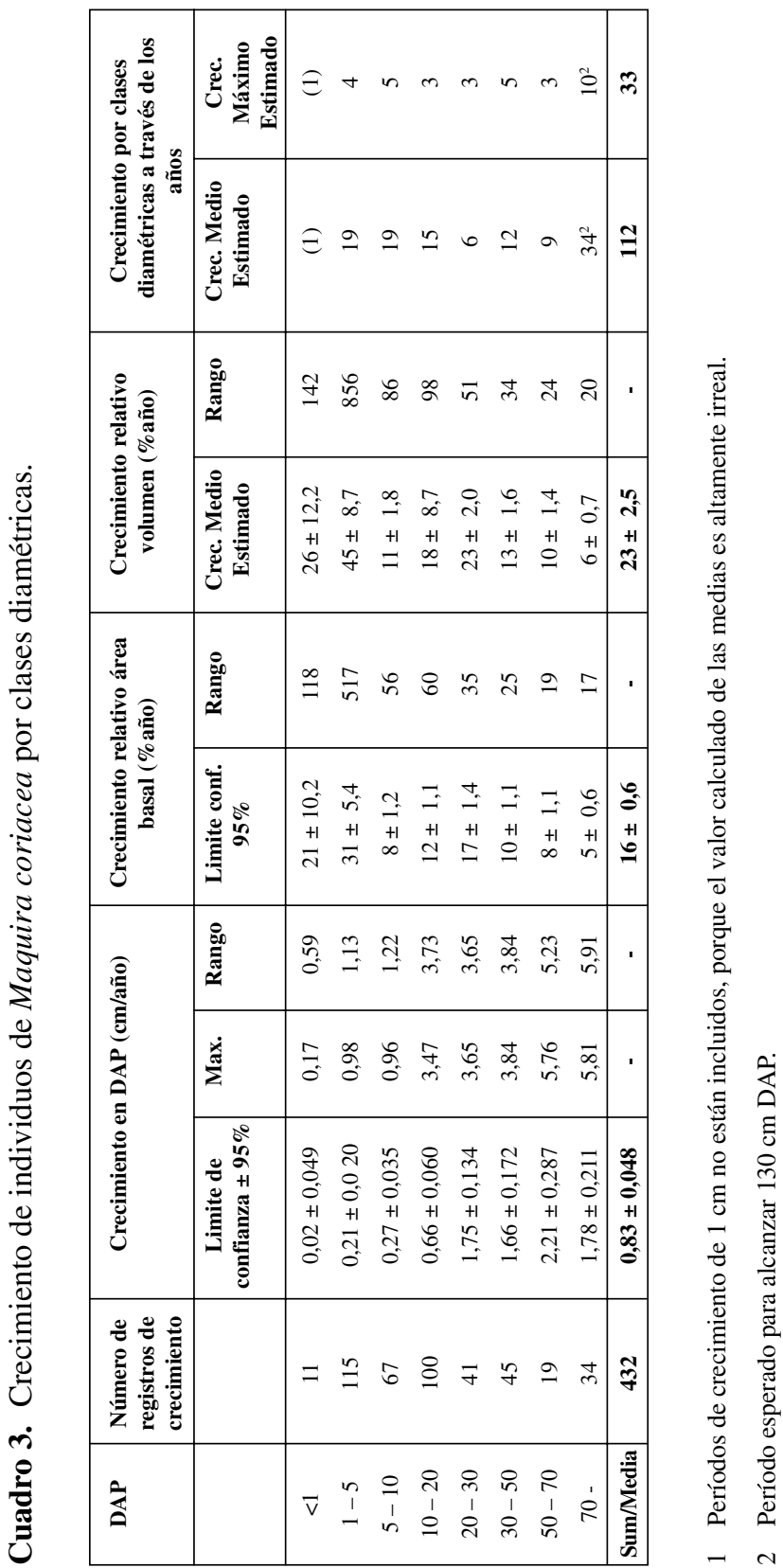


Figura 1

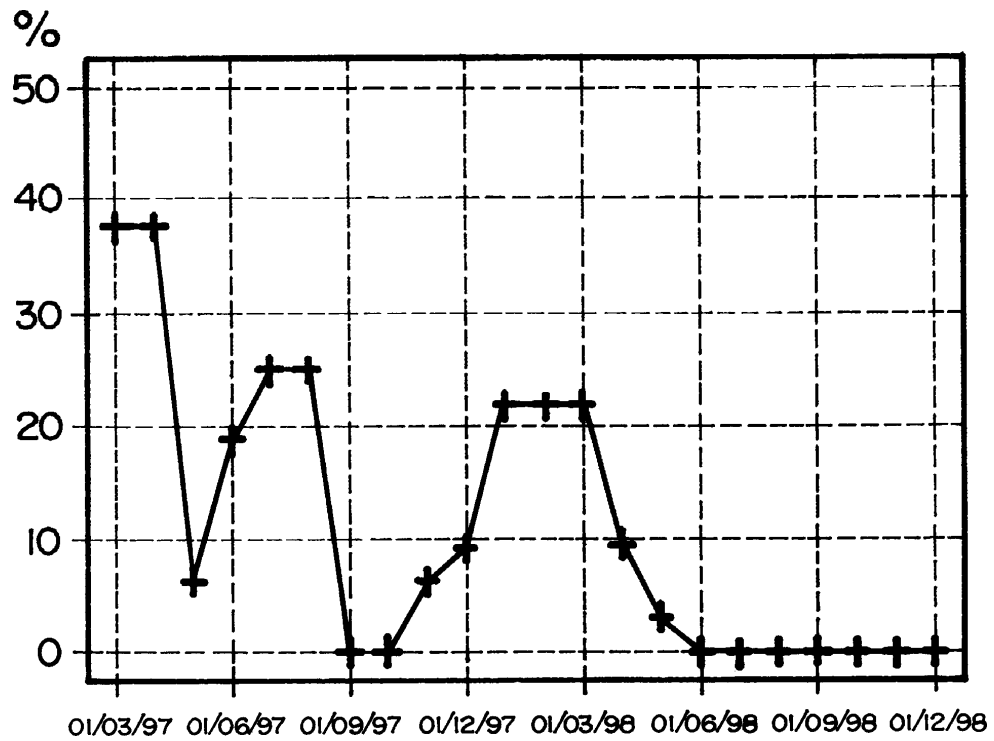

Fig. 1 Porcentaje de árboles madre entre 28 individuos de Maquira coriacea con DAP por encima de $42 \mathrm{~cm}$. 
Figura 2

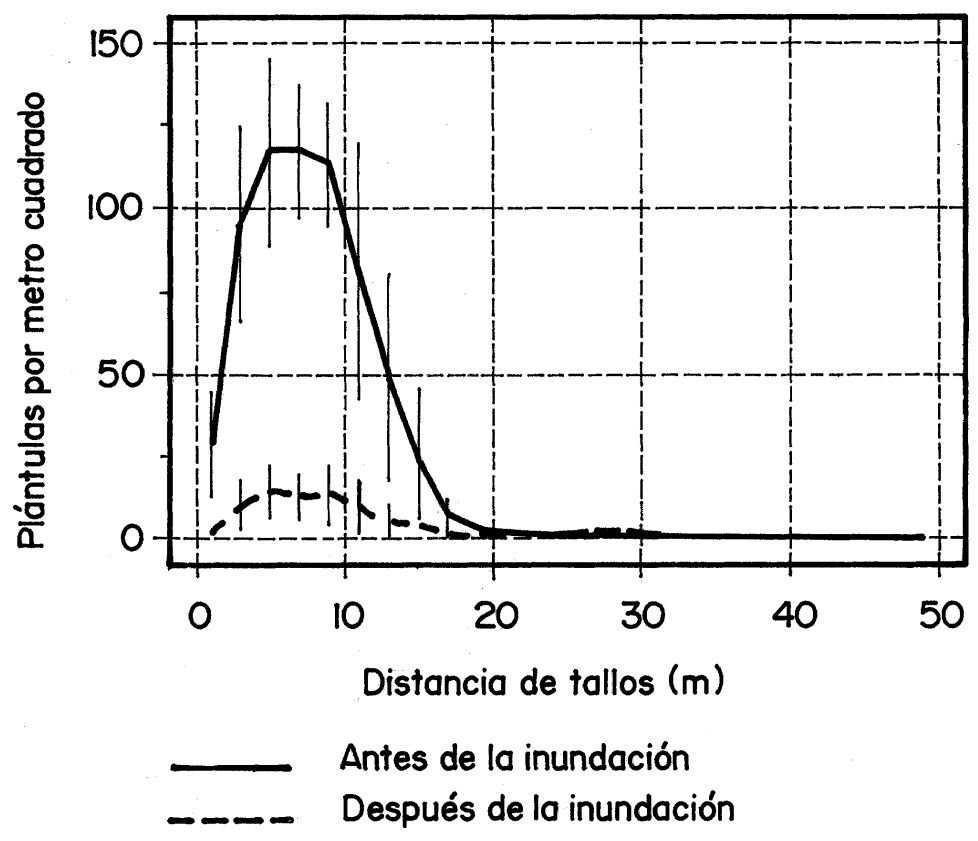

Fig. 2. Densidad de plántulas observadas en las parcelas localizadas a diferentes distancias de los tallos de cinco árboles madre aislados de Maquira coriacea. Promedio y límites al 95\% de confianza para la densidad de plántulas antes y después de la inundación. 


\section{Figura 3}

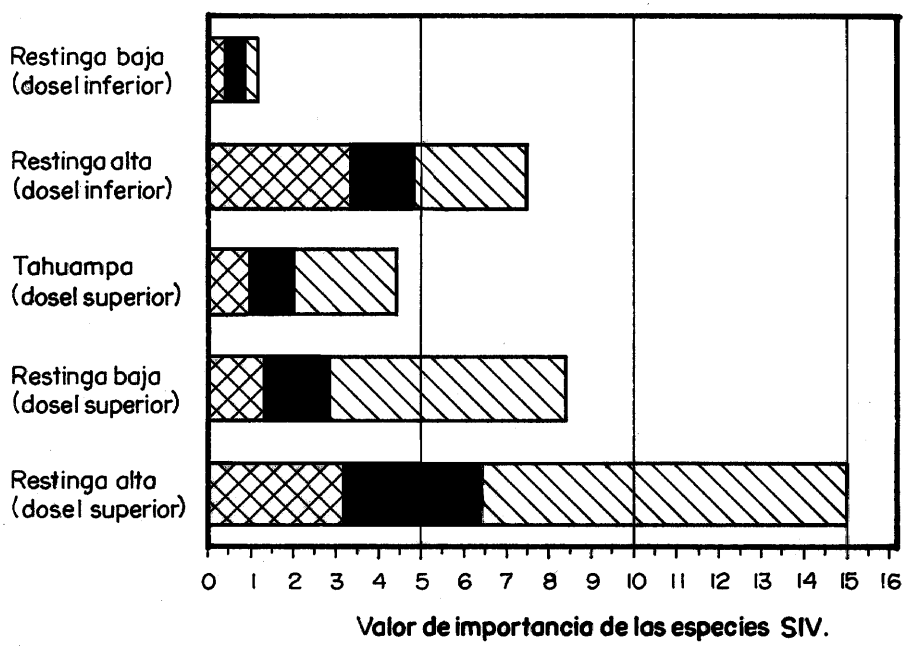

区 Deasidad relotiva Frecuencio relativo $\triangle \nabla$ Dominancio relativo

Fig. 3. Valores de importancia de las especies para Maquira coriacea en diferentes tipos de bosque y componentes de rodal. Por definición, la suma de los IVIs para todas las especies en un bosque o en un componente de rodal debe ser igual a 300. 
Figura 4

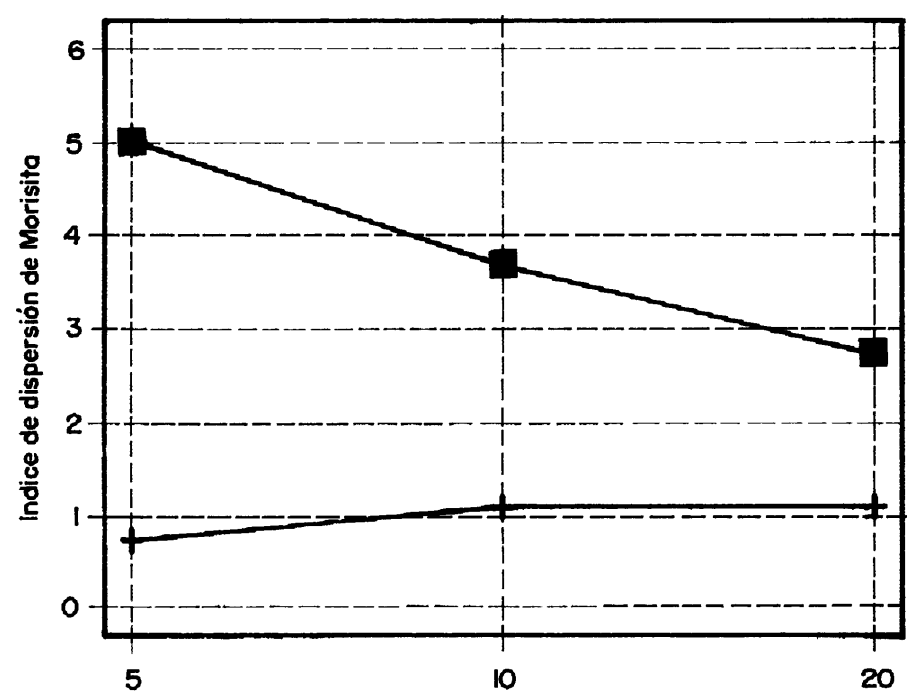

Superficie longitudinal de una parcela cuadrada

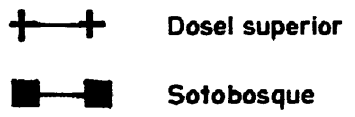

Fig. 4. Indice de dispersión de Morisita $\left(l_{\delta}\right)$ para los individuos de Maquira coriacea de estrato superior y sotobosque en cuadrantes de diferentes longitudes establecidos en la restinga alta. La abscisa está sobre una escala exponencial de acuerdo al área de la parcela muestra. 
Figura 5

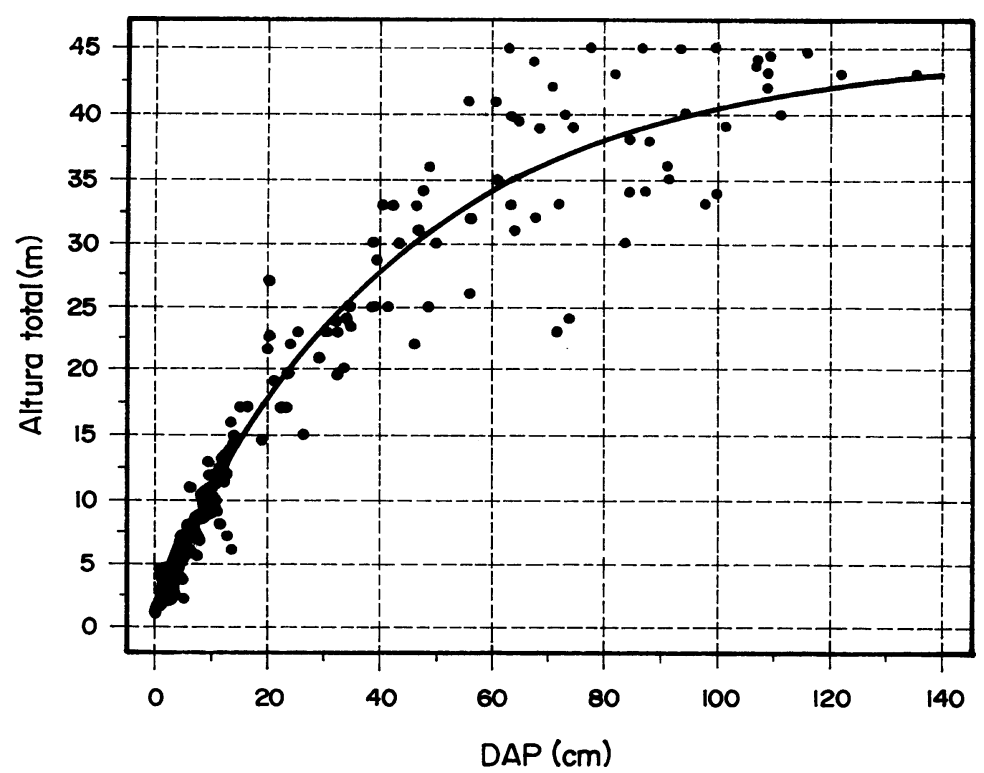

Fig. 5. Altura total versus DAP de Maquira coriacea: observaciones y correlaciones (modelo (2)). 

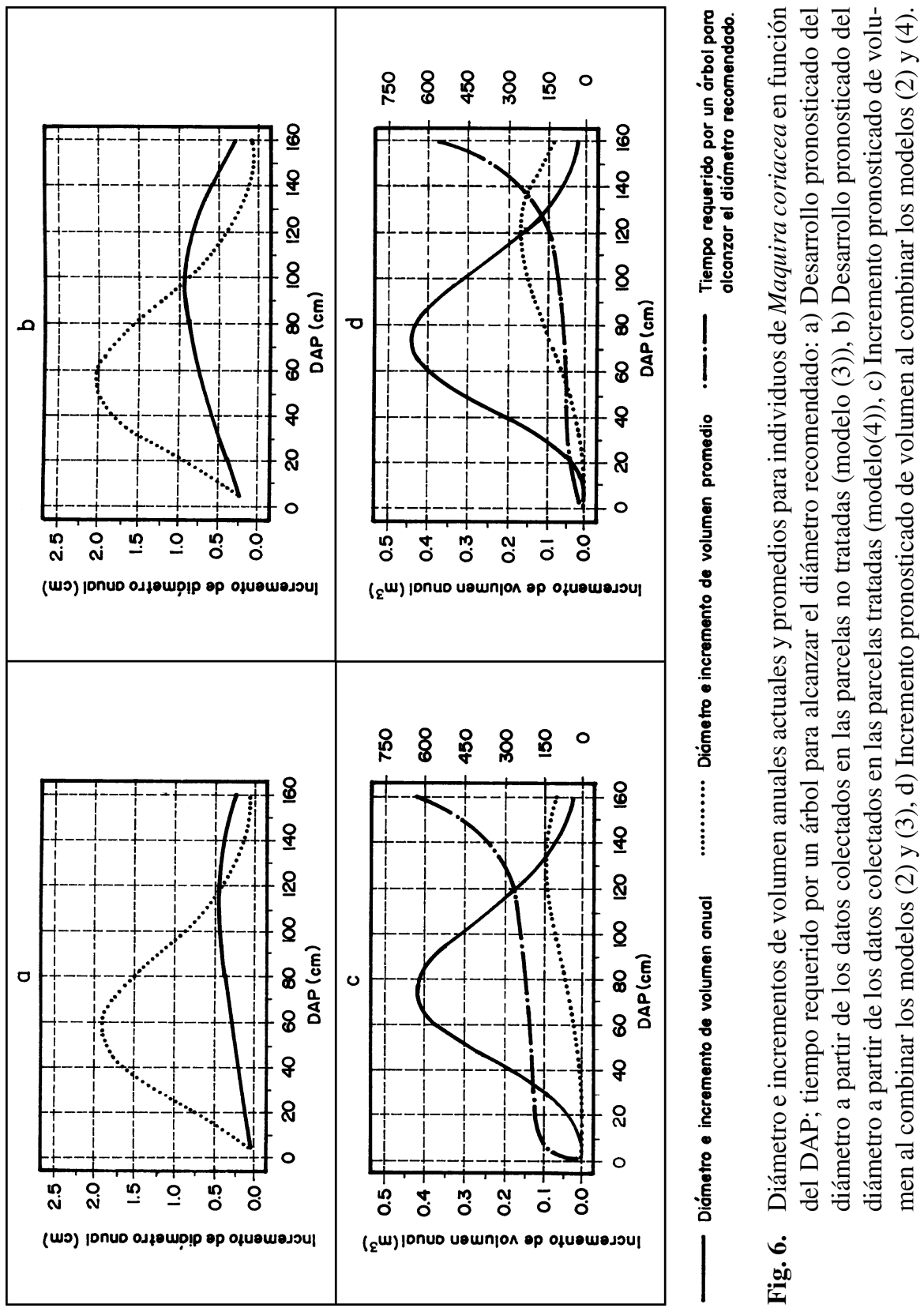